Research Paper

\title{
GLP-1 Treatment Improves Diabetic Retinopathy by Alleviating Autophagy through GLP-1R-ERK1/2-HDAC6 Signaling Pathway
}

\author{
Xiangsheng Cai ${ }^{1}$, 3* ${ }^{*}$ Jingjing $\mathrm{Li}^{1 *}$, Mingzhu Wang ${ }^{1}$, Miaoqin She ${ }^{1}$, Yongming Tang4, Jinlong Li ${ }^{1}$, Hongwei \\ $\mathrm{Li}^{1}$ and Hongxiang Hui ${ }^{1,2,3,4 \bowtie}$ \\ 1. School of Biotechnology, Southern Medical University, Guangzhou 510515, China; \\ 2. International Center for Metabolic Diseases, Southern Medical University, Guangzhou 510515, China; \\ 3. Dongguan SMU Metabolic Medicine R\&D Inc., Dongguan, Guangdong Province 523000, China; \\ 4. UCLA Center for Excellence in Pancreatic Disease, David Geffen School of Medicine, University of California, Los Angeles, California 90095, USA. \\ * These authors contributed equally to this work. \\ $\bowtie$ Corresponding author: Hongxiang Hui, Email: Huihongx@gmail.com; Hongwei Li, Email: lihw@smu.edu.cn.
}

(C) Ivyspring International Publisher. This is an open access article distributed under the terms of the Creative Commons Attribution (CC BY-NC) license (https://creativecommons.org/licenses/by-nc/4.0/). See http://ivyspring.com/terms for full terms and conditions.

Received: 2017.05.10; Accepted: 2017.08.07; Published: 2017.09.19

\begin{abstract}
Objective: Apoptosis and autophagy of retinal cells, which may be induced by oxidative stress, are tightly associated with the pathogenesis of diabetic retinopathy (DR). The autophagy induced by oxidative stress is considered as excessively stimulated autophagy, which accelerates the progression of DR. This study aims to investigate the protective effect of GLP-1 treatment on alleviating apoptosis and autophagy of retinal cells in type 2 diabetic rats and reveals its possible mechanism.

Methods: Type 2 diabetic rats were induced by fed with high sugar, high fat diet and followed with streptozotocin injection. GLP-1 was applied to treat the diabetic rats for one week after the onset of diabetes. The expressions of oxidative stress-related enzymes, retinal GLP-1R, mitochondria-dependent apoptosis- related genes, autophagy markers, and autophagy-associated pathway genes were studied by Western blotting or immunohistochemistry analysis.

Results: GLP-1 treatment reduced the levels of NOX3 and SOD2 in DR. The expression of BCL2 was increased, while the levels of caspase 3 and LC3B were reduced through GLP-1 treatment in DR. GLP-1 treatment restored the GLP-1R expression and decreased the levels of phosphorylated AKT and phosphorylated ERK1/2, which was accompanied with the reduction of the HDAC6 levels in DR.

Conclusions: GLP-1 treatment can alleviate autophagy which may be induced by oxidative stress; this protective effect is likely through GLP-1R-ERK1/2-HDAC6 signaling pathway.
\end{abstract}

Key words: retinopathy, GLP-1, oxidative stress, autophagy, HDAC6.

\section{Introduction}

Diabetes mellitus (DM) is an epidemic disease with extremely high morbidity [1]. There are more than 382 million people with diabetes worldwide, and the number of diabetic patients is dramatically increased [2]. Diabetic retinopathy (DR) is the most common diabetic complication which is a leading cause of vision loss in working population and the elderly [3,4]. It was reported that $90 \%$ of diabetic people were affected by DR [5-7]. In the early stage of $\mathrm{DR}$, it is characterized by a loss of pericytes and vascular endothelial cells, a breakdown of the blood-retinal barrier, vascular dysfunction and vascular-neuro inflammation [8]. Currently, the main strategies for treating DR are laser photocoagulation 
or drugs like anti-VEGF, anti-inflammatory molecules, and PKC inhibitors [9, 10]. However, all these strategies have undesirable side effects. Therefore, options for treating DR remain limited and more effective methods are urgently needed.

The pathogenesis of DR is complex, multiple processes are involved, including hyperglycemia, oxidative stress, advanced glycation end products (AGEs), protein kinase $\mathrm{C}$, inflammation and poly-ADP ribose polymerase (PARP) activation [11]. However, the mechanism of DR is still not entirely clear. Recently, growing evidences suggest that apoptosis and autophagy of retinal cells induced by oxidative stress are tightly associated with the pathogenesis of DR.

Previous studies showed that controlling DM could effectively inhibit the progression of DR $[12,13]$. Glucagon-like peptide-1 (GLP-1) is an endogenous insulin tropic peptide secreted by L-cells in response to food ingestion[14], which can regulate glucose metabolism, and is considered as a potential treatment for diabetes when administrated with effective concentrations $[15,16]$. Fan et al. showed that exendin-4, a GLP-1 receptor agonist, could alleviate retinal vascular leakage by protecting the blood-retinal barrier and reducing retinal vascular permeability in diabetic Goto-Kakizaki rats [17]. However, the molecular mechanism of GLP-1 treatment in diabetic retinopathy has not been clarified clearly.

As we known, diabetes will lead to increase of intracellular reactive oxygen species (ROS), subsequently inducing inflammation and apoptotic cell death $[18,19]$. Besides the insulin tropic effects of GLP-1 on insulin-secreting cells, increasing evidences indicate that GLP-1 has antioxidant effect [20-22]. Recent study indicated that GLP-1 improved $\mathrm{H}_{2} \mathrm{O}_{2}$-induced cellular injury [23]. Thus, the molecular mechanism of GLP-1 treatment in diabetic retinopathy may be tightly associated with its antioxidant effect.

ROS accumulation will induce autophagy [24], which plays a key role in the maintenance of normal cellular homeostasis [25, 26]. It is clear that basal autophagy can protect cells against oxidative stress by eliminating damaged intracellular materials [27]. However, it may cause autophagic death in retinal cells due to excessively stimulated autophagy. Russo et al. found that dysregulation of basal autophagy occurs under conditions of retinal transient ischemia which caused by dramatically elevated ROS induced through acutely increasing intraocular pressure in adult rats [28]. Herein, we hypothesize that GLP-1 may exert its antioxidant effect to protect the retinal cells through preventing the ROS-induced autophagy.
The mechanism of GLP-1treatment on preventing the ROS-induced autophagy remains unclear. It has been reported that epigenetic factor HDAC6 associates tightly with autophagy among all histone deacetylases [29]. In addition, HDAC6 regulates various cellular processes, including endocytosis, cell motility, cell migration, aggresome formation and autophagy etc., by deacetylating cytoplasmic proteins, such as heat shock protein 90 (HSP90), cortactin and a-tubulin [30].

In present study, we first investigated the effects of GLP-1on improving diabetic retinopathy in type 2 diabetic rats. We also revealed the possible mechanism of GLP-1 on alleviating apoptosis and autophagy in diabetic retinal cells.

\section{Materials and Methods}

\section{Reagents and Animals}

Streptozotocin (STZ) was purchased from Sigma (St. Louis, MO, USA). Formaldehyde and xylene were purchased from Guangzhou Chemical Reagent Factory (Guangzhou, China). Caspase3 antibody, LC3B antibody, p-Akt antibody, p-ERK1/2 and HDAC6 antibody were purchased from Cell Signaling Technology. Inc. (Danvers, Massachusetts, USA).

24 male SD rats (aged 6-8weeks, weighing $160-180 \mathrm{~g}$ ) were purchased from Experiment Animal Center of Southern Medical University (Guangzhou, China). All rats were housed in 12 hour light/dark cycle with providing water and food ad libitum. All animal experiments were carried out in accordance with the guidelines of the Animal Ethic Committee at Southern Medical University.

\section{Establishment of Type 2 Diabetic Rat Model}

24 rats were randomly divided into two groups: Diabetic group $(n=16)$ and the normal group $(n=8)$. The diabetic rats were established by fed with high fat and sugar $(20 \%$ sucrose, $10 \%$ lard, $2.5 \%$ cholesterol, $1 \%$ cholic acid and $66.5 \%$ conventional feed) for a month and intraperitoneally injected with STZ once with a dose of $40 \mathrm{mg} / \mathrm{kg}$ body weight. Animals were considered diabetic when glucose levels were higher than $16.7 \mathrm{mM}$ for 3 consecutive days (reference range, 5-8 $\mathrm{mM}$ ). The normal rats were fed with the standard rodent chow.

\section{Drug Treatment}

35 days after the onset of diabetes, the diabetic rats were randomly divided into two groups (each group, $n=8$ ), including diabetic retinopathy group (DR), GLP-1 treatment group (DR+GLP-1). In GLP-1 treatment group, GLP-1 was given via subcutaneous osmotic pump (30pmol/ $\mathrm{kg} / \mathrm{min})$ for 7 days in diabetic rats. Meanwhile, in DR and normal control 
groups, equal amount of normal saline were given to the rats for the same period.

After treatment, all rats were anesthetized by pentobarbital $(0.1 \mathrm{mg} / \mathrm{g}$ intraperitoneal injection) and sacrificed. Then, the eyeballs were removed and retina tissues were separated for HE staining and immunohistochemistry analysis.

\section{HE Staining}

Retinal tissues were selected randomly from each group. The samples were fixed with 10\% paraformaldehyde, decalcified with 20\% EDTA solution, dehydrated with ethanol by gradient, and embedded in paraffin. Afterwards, these specimens were cut into sections (4.0 5.0 $\mu \mathrm{m}$ thickness), stained with Hematoxylin-Eosin (HE) by a series of standard techniques. Histological changes were observed under light microscope.

\section{Immunoblot analysis}

Retinal tissues were collected and homogenized in RIPA buffer with PMSF. After centrifugation at $12000 \mathrm{~g}$ for $15 \mathrm{~min}$, the supernatant was collected and the protein concentrations were determined using the BCA (bicinchoninic acid) method [31]. Thirty micrograms of total cell lysate was applied to an SDS-PAGE $(10 \%)$ for separation and then transferred to a PVDF membrane. The membranes were then probed with primary antibodies (1:1000 dilution) at 4 ${ }^{\circ} \mathrm{C}$ overnight, followed with a secondary antibody (1:5000 dilution) conjugated with the horseradish peroxidase (HRP) for 1 hour at room temperature, and then visualized by enhanced chemiluminescence reagents (ECL; Pierce, Rockford, USA). Bands of interest were quantified by a densitometry, using Gel-Pro analysis software. The protein expression levels of rat glyceraldehyde-3-phosphate dehydrogenase (GAPDH) served as the house keeping control.

\section{Immunohistochemistry}

Retinal tissues were selected randomly from the remaining tissues of each group. After formalin-fixed and paraffin-embedded, specimens were cut into sections with 4 micron, dewaxed and hydrated, incubated with $3 \%$ hydrogen peroxide solution in dark at room temperature (RT) for 25 minutes, washed with PBS. Subsequently, the sections were incubated in 3\% BSA for 30 minutes at RT, and incubated with cleaved caspase 3 antibody (1:100), LC3B antibody (1:100), p-Akt antibody (1:100), p-ERK (1:100) and HDAC6 antibody (1:100) overnight, respectively. After that, the sections were washed 3 times with PBS, each time for 5 minutes, and stained by $\mathrm{DAB}$ using a secondary antibody kits. The staining time was controlled under the microscope and stopped by water washing. Finally, sections were re-stained by Harris hematoxylin for 3 minutes, and then washed, dehydrated, treated with xylene and coverslipped. The results were evaluated by light microscopy.

\section{Result}

\section{Blood glucose level}

As shown in table 1, blood glucose levels of all rats were normal before the diabetic model established. After that, the blood glucose levels of diabetic rats were significantly higher than that of the normal group $(23.8 \pm 2.2 \mathrm{mmol} / \mathrm{L}$ vs. $6.6 \pm 0.4$ $\mathrm{mmol} / \mathrm{L} ; \mathrm{p}<0.01)$, the blood glucose levels of rats of DM+GLP-1 group were also significantly higher than that of the normal group $(23.7 \pm 2.1 \mathrm{mmol} / \mathrm{L}$ vs. $6.6 \pm$ $0.4 \mathrm{mmol} / \mathrm{L} ; \mathrm{p}<0.01$ ).

After GLP-1 treatment for 1 week, GLP-1 reduced the blood glucose of the rats in the experimental group significantly, compared with the DM group $(18.2 \pm 1.1 \mathrm{mmol} / \mathrm{L}$ vs. $25.1 \pm 1.4 \mathrm{mmol} / \mathrm{L}$; $\mathrm{p}<0.01)$.

These results indicated that the type 2 diabetic rats were established successfully and GLP-1 administration was effective on controlling glucose.

Table 1. Blood glucose levels of rats in different experimental groups

\begin{tabular}{lllll}
\hline Group & $\mathrm{n}$ & \multicolumn{3}{l}{ Blood glucose $(\mathrm{mmol} / \mathrm{L})$} \\
\cline { 3 - 5 } & & $\begin{array}{l}\text { Before model } \\
\text { establishment }\end{array}$ & Before treatment & After treatment \\
\hline Normal & 8 & $6.6 \pm 0.2$ & $6.6 \pm 0.4$ & $6.9 \pm 0.7$ \\
DR & 8 & $6.9 \pm 0.7$ & $23.8 \pm 2.2^{* *}$ & $25.1 \pm 1.4^{* *}$ \\
DR+GLP-1 & 8 & $6.7 \pm 0.3$ & $23.7 \pm 2.1^{* *}$ & $18.2 \pm 1.1 \# \#$ \\
\hline
\end{tabular}

Annotation: Compared with normal group before treated, ${ }^{* *} \mathrm{P}<0.01$; Compared with DR group after treated, ${ }^{\#} \mathrm{P}<0.01$.

\section{The pathomorphology observation of retinal tissues}

Under an optical microscope, the retinal tissues of HE-stained showed that the thicknesses of columnar and cone photoreceptors, OPL (outer nuclear layer), INL (inner nuclear layer), IPL (inner plexiform layer) and GCL (retinal ganglion cell layer) were all significantly reduced in the DR group compared with the control group (Fig. 1). But the retinal morphology condition was improved effectively after treated with GLP-1 in DR rats. In addition, compared to other groups, the retina of normal group had a smoother surface, and RGCs (retinal ganglion cells), INL (inner nuclear layers) and ONL (outer nuclear layers), rod cells and cone cells were all arranged more regularly. 

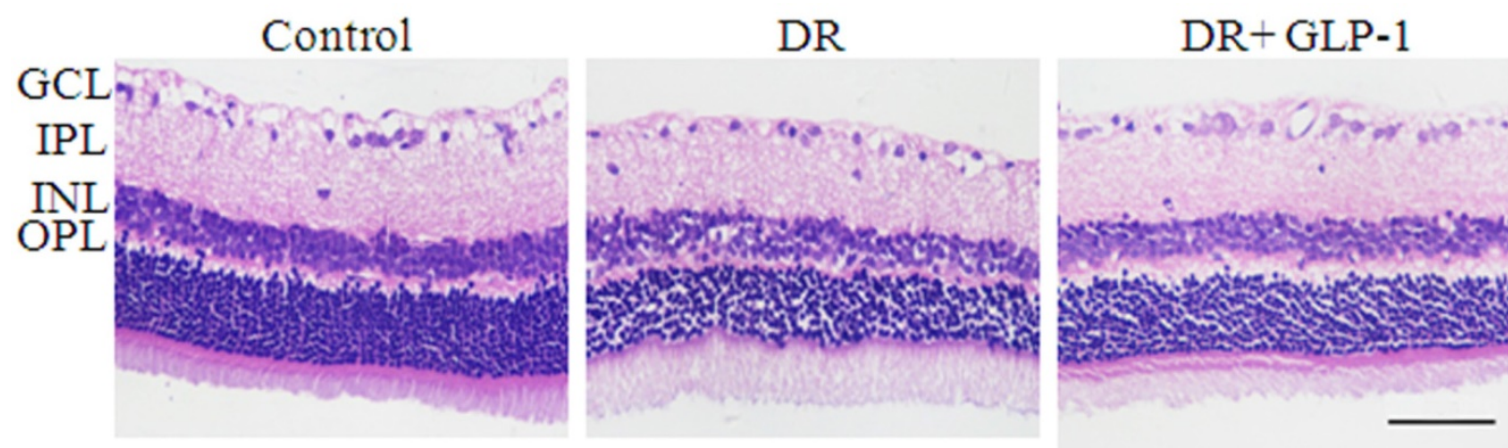

Figure 1. Photomicrographs of retinas from rats with hematoxylin-eosin (HE) staining of three groups: control, DR and DR+GLP-1. The scale bar represents $50 \mu \mathrm{m}$.

A

\section{Control}

DR

$\mathrm{DR}+\mathrm{GLP}-1$
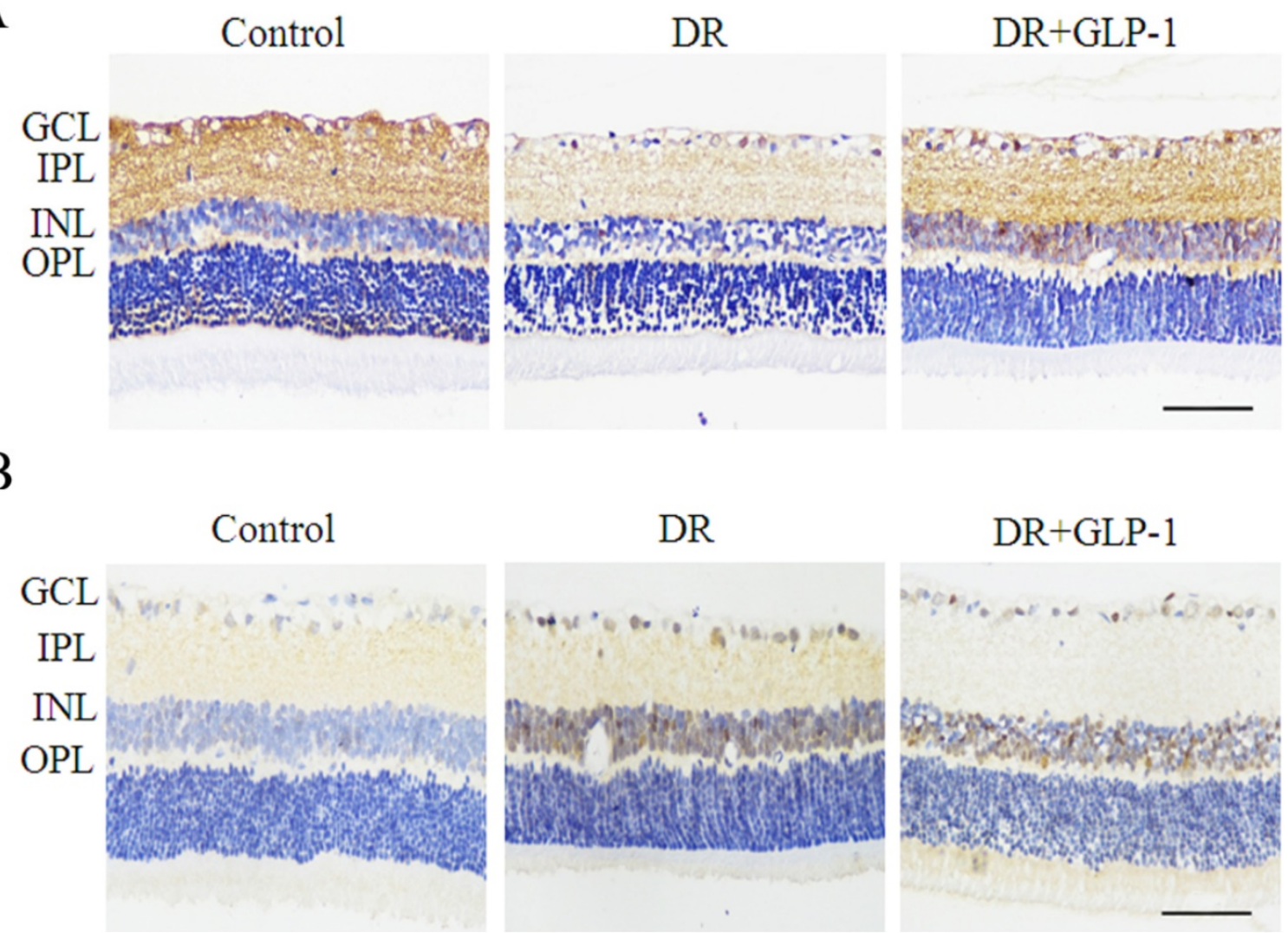

Figure 2. Immunostaining of $B C L 2$ and caspase- 3 in rat retinas of three groups: control, $D R$ and $D R+G L P-1$. (A) Levels of $B C L 2$ were significantly reduced in $D R$ and increased after GLP-1 treatment. (B) Levels of caspase-3 were significantly increased in DR and reduced after GLP-1 treatment. The scale bar represents 50 $\mu m$.

\section{GLP-1 alleviates apoptosis and autophagy in retinal cells in diabetic rats}

We investigated the effect of GLP-1 on apoptosis of diabetic retinal cells. The levels of BCL2 and caspase 3, two mitochondria-dependent apoptosis-related genes, were examined in this study (Fig. 2). The levels of BCL2 were decreased dramatically in DR group, but upregulated after GLP-1 treatment. The levels of caspase-3 were increased notably in DR group, but reduced after GLP-1 treatment.

Then, the effects of GLP-1 on autophagy of diabetic retinal cells were investigated through
Western blot analysis. As shown in Fig.3A, LC3II/I levels were significantly increased in retinal cells of diabetic rats compare with those of healthy rats $(p<0.01)$, which were attenuated by GLP-1 treatment $(\mathrm{p}<0.01)$.

Furthermore, we examined changes in the levels of LC3B in retinas through immunohistochemistry (Fig.3B). Levels of LC3B in retinas were significantly increased in the DR group compared with those in the control group, which was attenuated through GLP-1 treatment. These results suggested that GLP-1 treatment could effectively suppress apoptosis and autophagy of retinal cells in diabetic rats. 
A
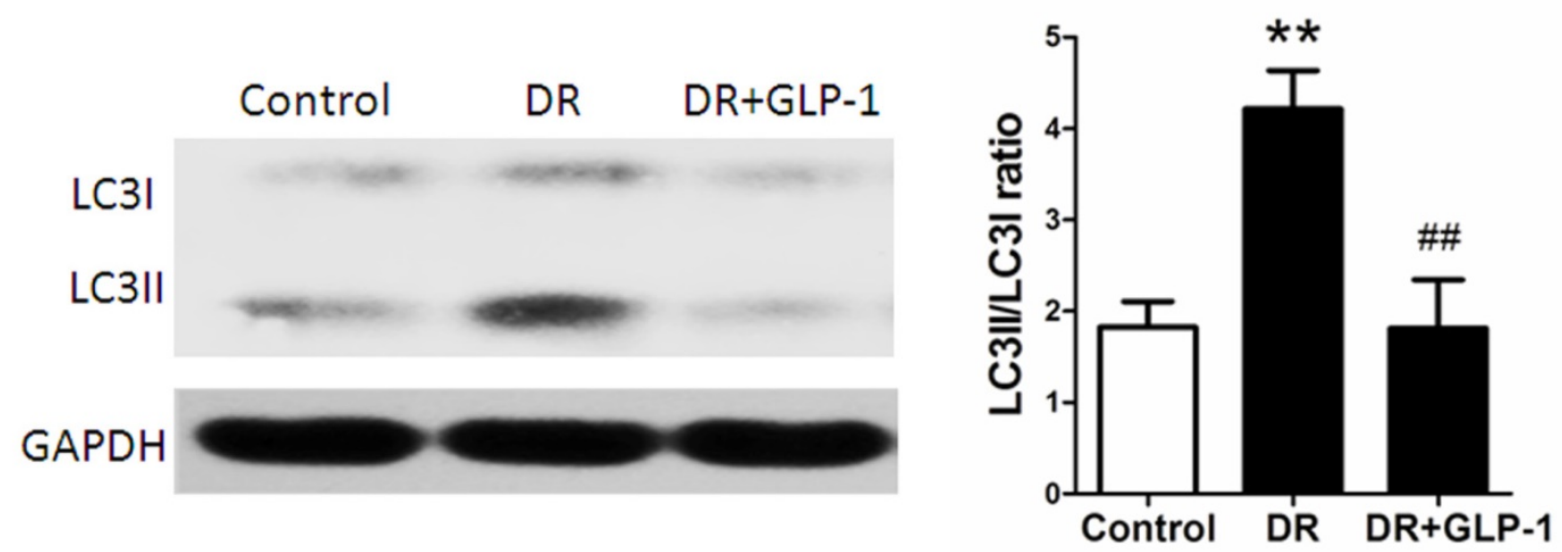

$\mathrm{B}$

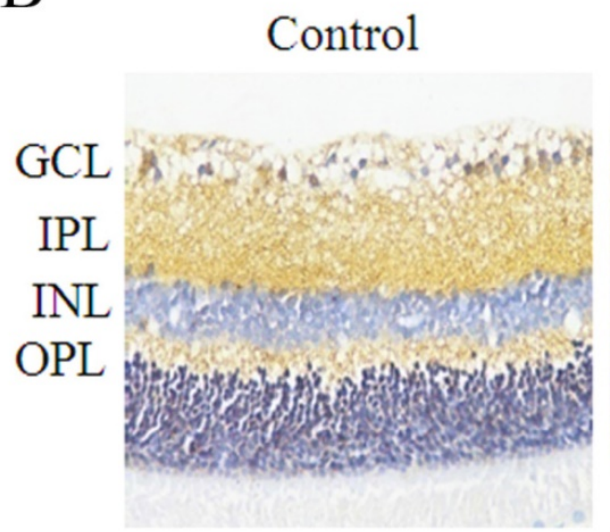

DR

$\mathrm{DR}+\mathrm{GLP}-1$

Figure 3. Reduction of LC3B protein expression through GLP-1 treatment. (A) Western blot analysis of retinal LC3B expression in three groups. (B) Immunohistochemistry of retinal GLP-IR in three groups. Data are expressed as the means \pm SEM. Statistical analyses were performed by one-way ANOVA followed by Tukey's test. *** $<0.01$ versus the normal group, $\#+0.01$ versus the $D R$ group. The scale bar represents $50 \mu \mathrm{m}$.

\section{GLP-1 treatment alleviates oxidative stress in retinas of diabetic rats}

As the oxidative stress is a crucial mechanism causing the occurrence of apoptosis and autophagy, we assess the changes in oxidative stress of diabetic rats. The levels of SOD2 and NOX3, which were oxidative stress-related enzymes, were investigated to evaluate the effect of GLP-1 on alleviating oxidative stress of retinal cells of T2D rats. As shown in Fig.4, immunohistochemistry results showed that the levels of SOD2 and NOX3 were upregulated in DR group compared with those of normal group, implying an oxidative stress response in diabetic rats. The levels of SOD2 and NOX3 were decreased in DR+GLP-1 group compared with those of DR group, which indicated that GLP-1 treatment could alleviate the oxidative stress in diabetic rats.

\section{GLP-1 treatment restored the GLP-1R expression in diabetic rats}

It is widely accepted that GLP-1 exerts its effect through GLP-1R. We hypothesize that GLP-1 alleviating apoptosis and autophagy through GLP-1R. Thus, we examined the changes in GLP-1R expression in both retinas of healthy and diabetic rats treated with or without GLP-1. As shown in Fig.5A, GLP-1R level was decreased in DR group compared with those of the normal control group $(p<0.01)$, but was restored under GLP-1 treatment $(\mathrm{p}<0.01)$.

The changes in GLP-1R expression indicated by immunohistochemistry analysis were consistent with those shown by western blot analysis. GLP-1R was expressed in the inner layers of the retina, primarily in the INL and ganglion cells. As shown in Fig.5B, GLP-1R expression was significantly decreased in retinal cells of diabetic rats compare with those of healthy rats, while GLP-1 treatment notably restored the expression of GLP-1R. 
A

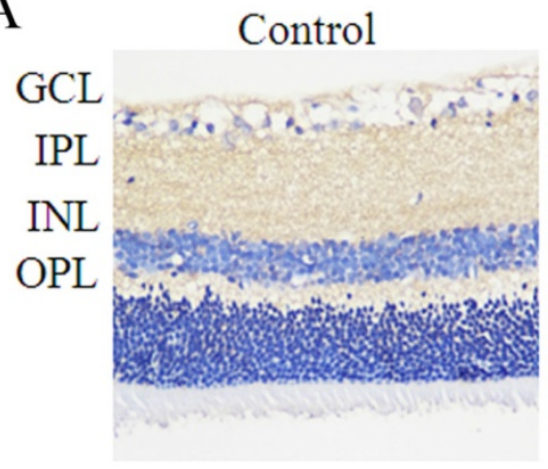

$\mathrm{DR}$

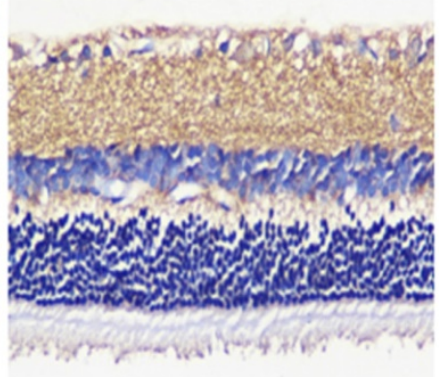

$\mathrm{DR}+\mathrm{GLP}-1$

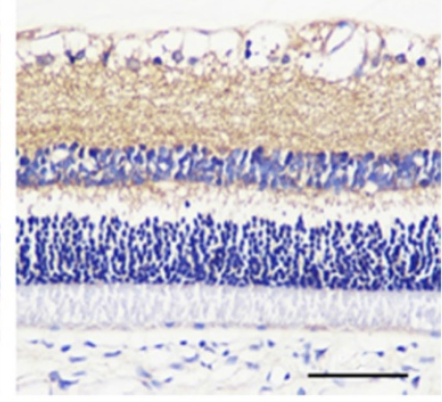

$\mathrm{DR}+\mathrm{GLP}-1$

B

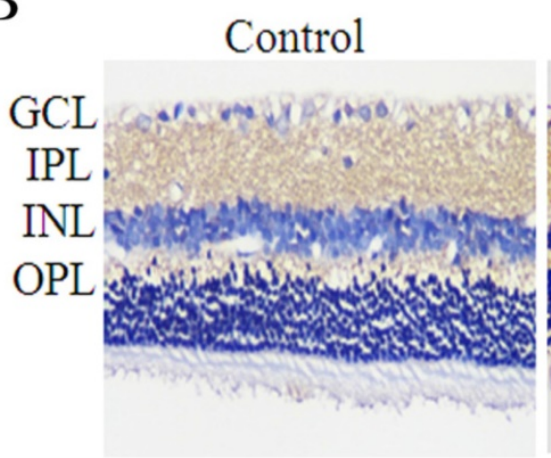

$\mathrm{DR}$

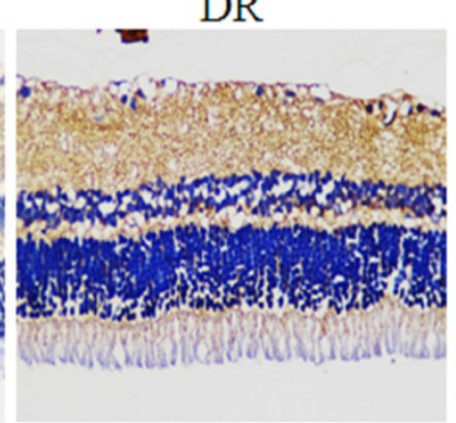

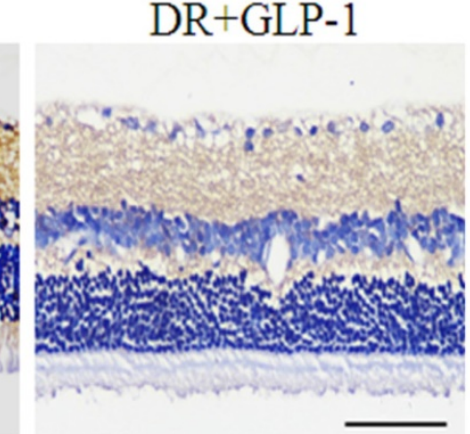

Figure 4. Detection of SOD2 and NOX3 in rat retinas of three groups: control, DR and DR+GLP-1. Levels of SOD2 (A) and NOX3 (B) were dramatically increased in DR and reduced after GLP-1 intervention. The scale bar represents $50 \mu \mathrm{m}$.

A
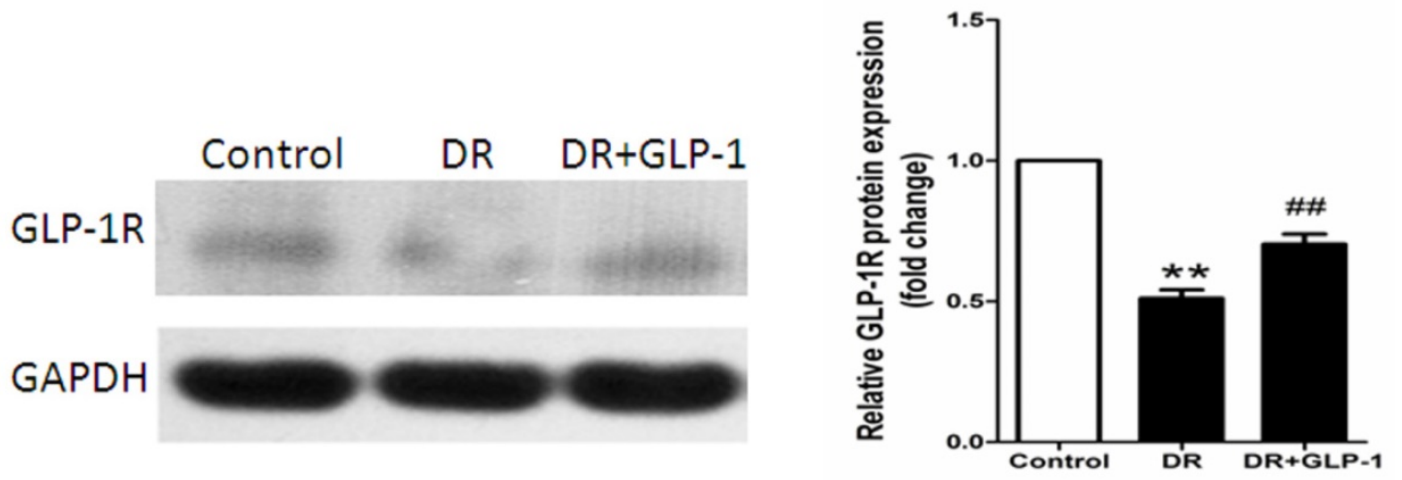

B

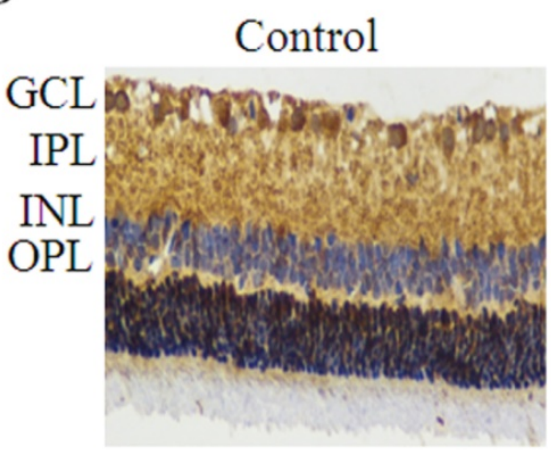

$\mathrm{DR}$

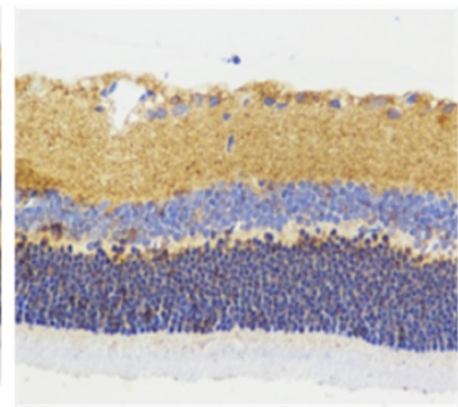

$\mathrm{DR}+\mathrm{GLP}-1$

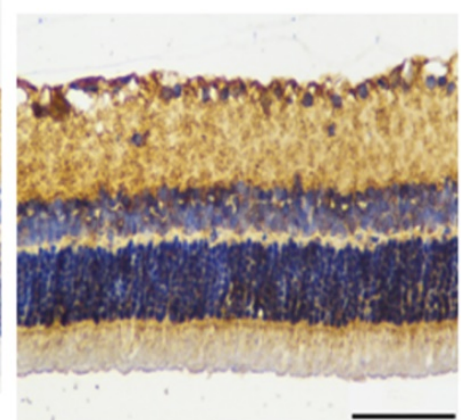

Figure 5. Maintenance of GLP-1R protein expression by GLP-1 stimulation. (A) Western blot analysis of retinal GLP-1R expression in three groups. (B) Immunohistochemistry of retinal GLP-IR in three groups. Data are expressed as the means \pm SEM. Statistical analyses were performed by one-way ANOVA followed by Tukey's test. ${ }^{*} * \mathrm{P}<0.01$ versus the normal group, \# $\mathrm{P}<0.01$ versus the $\mathrm{DR}$ group. The scale bar represents $50 \mu \mathrm{m}$. 


\section{GLP-1 alleviates apoptosis and autophagy of retinal cells via AKT/ERK pathway}

To reveal the possible mechanism underlying the effect of GLP-1 on alleviating apoptosis and autophagy of diabetic retinal cells, we investigated the AKT/ERK pathway in this study, which has been previous reported to associate with apoptosis and autophagy tightly. As shown in Fig.6, the immunohistochemistry results indicated that the levels of phosphorylated Akt and ERK1/2 were increased significantly in DR group compared with normal group, especially in the sections of GCL, INL and OPL of retina tissues, which were attenuated after GLP-1 treatment.

\section{GLP-1 alleviates autophagy through modulating HDAC6 expression}

As final effectors of ERK-MAPK pathway, ERK1/2 was reported to interact with HDAC6 in vivo, which play a key role in oxidative stress response and autophagy. Herein, HDAC6 expression was investigated through Immunoblot analysis. As shown in Fig.7A, HDAC6 level was significantly increased in retinal cells of diabetic rats compare with those of healthy rats $(p<0.01)$, while GLP-1 treatment notably reduced the expression of HDAC6 $(\mathrm{p}<0.05)$.

The changes in HDAC6 expression indicated by immunohistochemistry analysis were consistent with those shown by western blot analysis. As shown in Fig.7B, HDAC6 expression was significantly increased in retinal cells of diabetic rats compare with those of healthy rats, while GLP-1 treatment notably reduced the expression of HDAC6.

\section{Discussion}

Diabetic retinopathy is the leading cause of blindness in working population and the elderly [3, 4]. It is well known that retinal degeneration in proliferative diabetic retinopathy is irreversible. In addition, current treatments for DR of advanced stages are linked to notable adverse effects $[9,10]$. Therefore, new approaches for DR treatment at early stages are needed.

It is clear that type 2 diabetes mellitus generates chronic and acute toxicity to vascular endothelium in patients, including dyslipidemia, hyperglycemia, and increased oxidative stress [32]. Oxidant injury or inflammation induced by diabetes often cause complications such as neuropathy, nephropathy and retinopathy.

A
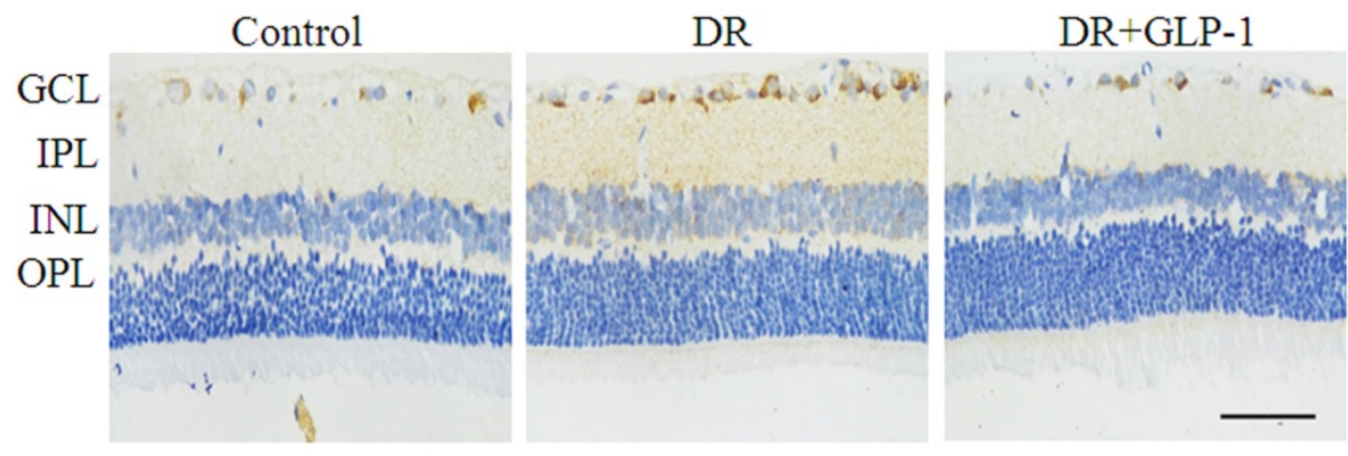

B

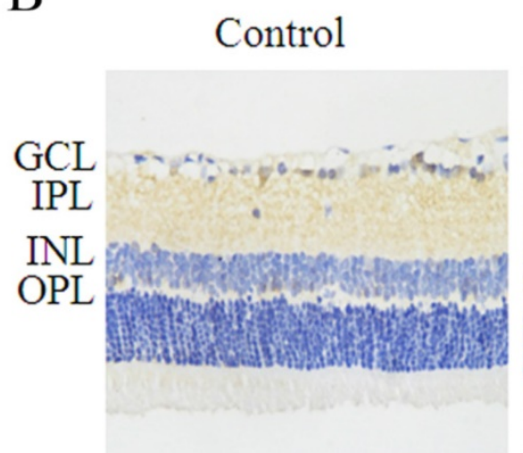

DR

$\mathrm{DR}+\mathrm{GLP}-1$
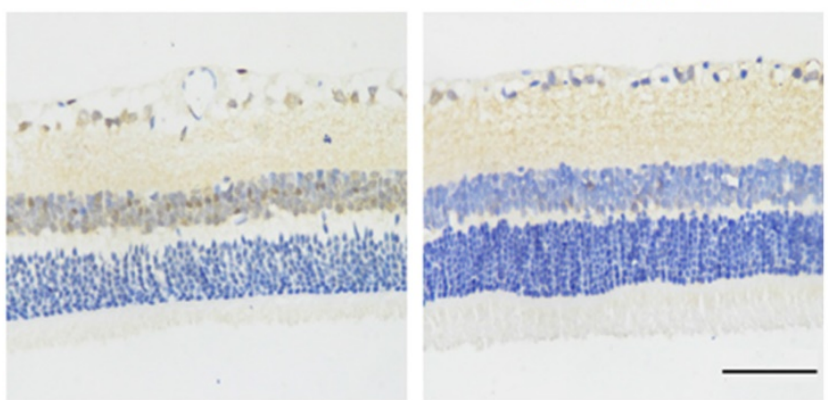

Figure 6. Reduction of $\mathrm{p}-\mathrm{Akt}$ and $\mathrm{p}$-ERK $1 / 2$ by GLP-1 treatment in diabetic rat retinas. Levels of $\mathrm{p}-\mathrm{Akt}(\mathrm{A})$ and $\mathrm{p}-\mathrm{ERK} 1 / 2$ (B) were significantly upregulated in DR and decreased after GLP-1 treatment. The scale bar represents $50 \mu \mathrm{m}$. 
A
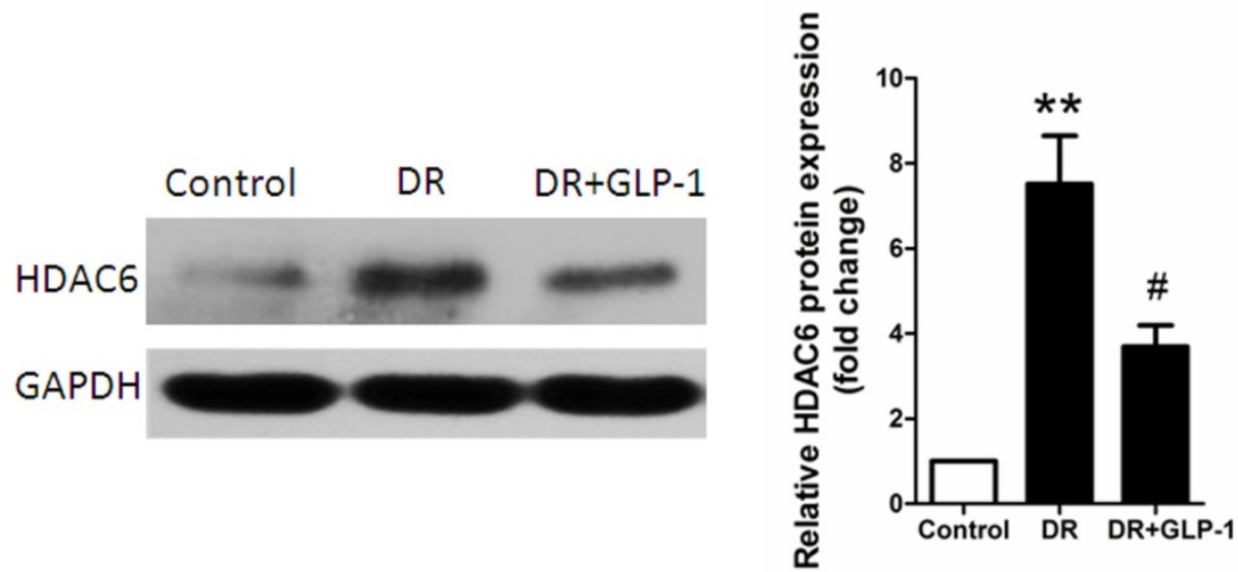

$\mathrm{B}$

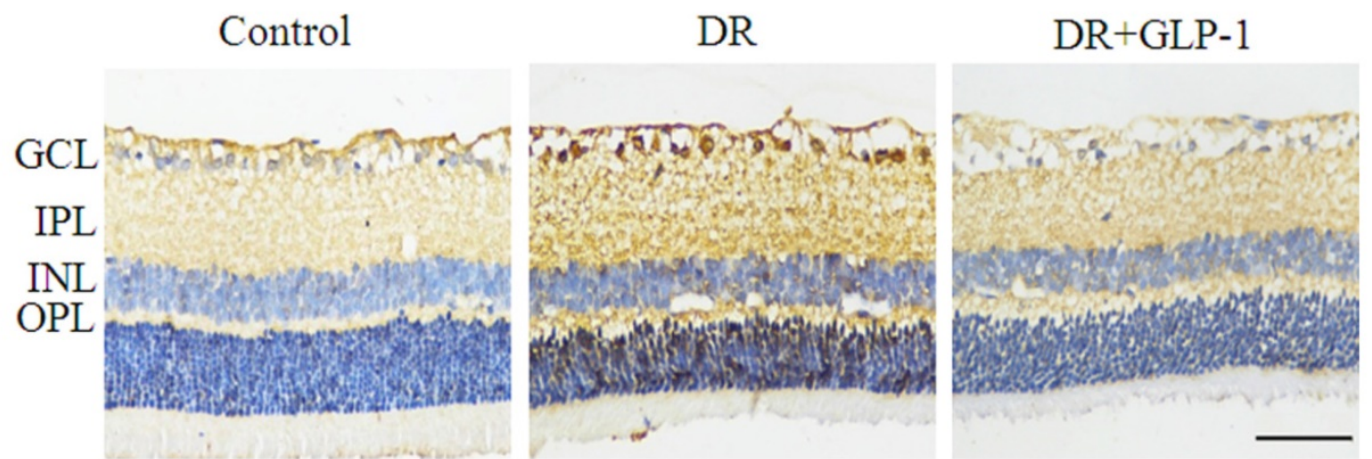

Figure 7. Reduction of HDAC6 protein expression by GLP-1 treatment in diabetic rat retinas. (A) Western blot analysis of retinal HDAC6 expression in three groups. (B) Immunohistochemistry analysis of retinal HDAC6 in three groups. Data are expressed as the means \pm SEM. Statistical analyses were performed by one-way ANOVA followed by Tukey's test. ${ }^{*} \mathrm{P}<0.01$ versus the normal group, \# $\mathrm{P}<0.05$ versus the $\mathrm{DR}$ group. The scale bar represents $50 \mu \mathrm{m}$.

Excessive ROS generation may accelerate pathophysiological progression in diabetes, which plays a pivotal role of oxidative stress in the onset and development of diabetes complications [24]. In present study, the NOX3 and SOD2, which were considered as the indicators of oxidative stress response in vivo, were investigated through immunohistochemistry analysis. Our results showed that GLP-1 could reduce the levels of the NOX3 and SOD2 induced by oxidative stress in T2D rats. Thus, GLP-1 is beneficial to alleviate the oxidative stress in DR.

As we known, oxidant injury contributes to a major mechanism causing apoptosis in diabetes. The mitochondria-dependent apoptosis pathway plays a key role in diabetic-induced retinal cell apoptosis. In our study, the reduction of BCL2 levels and increase of caspase 3 levels indicated an occurrence of apoptosis in T2D rat retinas, while GLP-1 treatment could effectively alleviate apoptosis in DR.

On the other hand, autophagy is a potent adaptive mechanism, which protects cells against oxidative stress-caused injury by the lysosome-mediated degradation of damaged or dysfunctional organelles and protein aggregates [25,
26]. However, excessively stimulated autophagy in retinal cells may cause autophagic death, which may lead to advanced stages of DR. Therefore, preventing occurrence of excessively stimulated autophagy at early stages may be benefit to DR treatment. Our results showed that autophagy pathway was activated in diabetic retinas, which was mainly due to oxidative stress, might possibly become excessively stimulated autophagy and lead to autophagic death. This autophagy effect was significantly alleviated by GLP-1 treatment. To our best knowledge, it is the first report on the effect of GLP-1 on alleviating excessively stimulated autophagy of retinal cells.

Up to date, the mechanism underlying the effect of GLP-1 treatment on alleviating apoptosis and excessively stimulated autophagy remains not clear. Previous study indicated that GLP-1exerted its function through GLP-1R. In line with our study, GLP-1R was diffusely distributed in the retina. The GLP-1R expression was decreased in diabetic retinas, while GLP-1 treatment could maintain the GLP-1R levels. In addition, previous study showed that autophagy was a key cellular process against oxidative stress mediated mitochondrial injury [33]. Our results showed that oxidative stress might 
promote autophagy in retinas of diabetic rats through activation of Akt and ERK signaling pathway. The AKT and ERK were significantly activated in the type 2 diabetic rats, and these tendencies were suppressed through GLP-1 treatment.

As final effectors of ERK-MAPK pathway, ERK1/2 was reported to interact with HDAC6 in vivo [34]. One study indicated that overexpression of HDAC6 in HEK293T cells increased the phosphorylation level of ERK1/2 [35]. In our study, in DR group, ERK1/2 phosphorylation was increased accompanied with upregulation of the levels of HDAC6, which were attenuated by GLP-1treatment.

Epigenetic factor HDAC6 has been reported to associate tightly with cell autophagy among all histone deacetylases. Previous study revealed that HDAC6 mediated retrograde transport of inclusion bodies containing aggregate to be degraded by autophagy through deacetylating a-tubulin. In line with our study, HDAC6 levels were increased in retinas of diabetic rats compared with healthy rats, which were associated with autophagy induced by oxidative stress injury, was attenuated by GLP-1treatment. To our best knowledge, it is the first report that the protective effects of GLP-1against DR may be through modulating HDAC6 expression.

In conclusion, we shed light on the mechanisms underlying the protective effects of GLP-1 against diabetic retinopathy in T2D rats. Our results suggest that GLP-1 treatment alleviates oxidative stress in DR. GLP-1 treatment alleviates apoptosis and autophagy of retinal cells in diabetic rats, the anti-autophagic effect of GLP-1 on retinal cells may be through GLP-1R-ERK1/2-HDAC6 signaling pathway. These findings reinforce the effect of GLP-1 in diabetic retinopathy. Thus, it may be an effective approach to treat DR by GLP-1.

\section{Acknowledgements}

This work was supported by grants from the National 973 Projects (no. 2011CB504006), the Songshan Lake Science and Technology Fund (no. 2010B025 \& no. 2010B026), the Guangdong Science and Technology Fund (no. 2010B090400041) and the Doctoral Program of Higher Education of the Ministry of Education (no. 20104433110014).

\section{Competing Interests}

The authors have declared that no competing interest exists.

\section{References}

1. Shaw JE, Sicree RA, Zimmet PZ: Global estimates of the prevalence of diabetes for 2010 and 2030. Diabetes Res Clin Pract. 2010; 87:4-14.
2. Guariguata L, Whiting DR, Hambleton I, Beagley J, Linnenkamp U, Shaw JE: Global estimates of diabetes prevalence for 2013 and projections for 2035. Diabetes Res Clin Pract. 2014; 103:137-149.

3. Funatsu H, Yamashita H: [Molecular biology in development and progression of diabetic retinopathy]. Nihon Rinsho. 2002; 60 Suppl 10:162-166.

4. Wilkinson CP, Ferris FL, 3rd, Klein RE, Lee PP, Agardh CD, Davis M, Dills D, Kampik A, Pararajasegaram R, Verdaguer JT, Global Diabetic Retinopathy Project G: Proposed international clinical diabetic retinopathy and diabetic macular edema disease severity scales. Ophthalmology. 2003; 110:1677-1682.

5. Glover SJ, Burgess PI, Cohen DB, Harding SP, Hofland HW, Zijlstra EE, Allain TJ: Prevalence of diabetic retinopathy, cataract and visual impairment in patients with diabetes in sub-Saharan Africa. Br J Ophthalmol. 2012; 96:156-161.

6. Sundling V, Platou CG, Jansson RW, Bertelsen G, Wollo E, Gulbrandsen P: Retinopathy and visual impairment in diabetes, impaired glucose tolerance and normal glucose tolerance: the Nord-Trondelag Health Study (the HUNT study). Acta Ophthalmol. 2012; 90:237-243.

7. Jin J, Min H, Kim SJ, Oh S, Kim K, Yu HG, Park T, Kim Y: Development of Diagnostic Biomarkers for Detecting Diabetic Retinopathy at Early Stages Using Quantitative Proteomics. J Diabetes Res. 2016; 2016:6571976.

8. Velasquez T, Mackey G, Lusk J, Kyle UG, Fontenot T, Marshall P, Shekerdemian LS, Coss-Bu JA, Nishigaki A, Yatabe T, et al: ESICM LIVES 2016: part three : Milan, Italy. 1-5 October 2016. Intensive Care Med Exp. 2016; $4: 28$.

9. Liu N, Zhao N, Chen L, Cai N: Survivin contributes to the progression of diabetic retinopathy through HIF-1alpha pathway. Int J Clin Exp Pathol. 2015; 8:9161-9167.

10. Dorrell M, Uusitalo-Jarvinen H, Aguilar E, Friedlander M: Ocular neovascularization: basic mechanisms and therapeutic advances. Surv Ophthalmol. 2007; 52 Suppl 1:S3-19.

11. Wan TT, Li XF, Sun YM, Li YB, Su Y: Recent advances in understanding the biochemical and molecular mechanism of diabetic retinopathy. Biomed Pharmacother. 2015; 74:145-147.

12. Antonetti DA, Klein R, Gardner TW: Diabetic retinopathy. N Engl J Med. 2012; 366:1227-1239.

13. Mohamed $Q$, Gillies MC, Wong TY: Management of diabetic retinopathy: a systematic review. JAMA. 2007; 298:902-916.

14. Baggio LL, Drucker DJ: Biology of incretins: GLP-1 and GIP. Gastroenterology. 2007; 132:2131-2157.

15. Malendowicz LK, Macchi C, Nussdorfer GG, Nowak KW, Zyterska A, Ziolkowska A: Effects of prolonged exendin-4 administration on entero-insular axis of normal and streptozotocin-induced diabetic rats. Int J Mol Med. 2003; 11:763-766.

16. Nauck MA, Kleine N, Orskov C, Holst JJ, Willms B, Creutzfeldt W: Normalization of fasting hyperglycaemia by exogenous glucagon-like peptide 1 (7-36 amide) in type 2 (non-insulin-dependent) diabetic patients. Diabetologia. 1993; 36:741-744.

17. Fan YC, Liu K, Wang QP, Ruan YY, Ye W, Zhang Y: Exendin-4 alleviates retinal vascular leakage by protecting the blood-retinal barrier and reducing retinal vascular permeability in diabetic Goto-Kakizaki rats. Experimental Eye Research. 2014; 127:104-116.

18. Chang YC, Chuang LM: The role of oxidative stress in the pathogenesis of type 2 diabetes: from molecular mechanism to clinical implication. American Journal of Translational Research. 2010; 2:316-331.

19. Naudi A, Jove M, Ayala V, Cassanye A, Serrano J, Gonzalo H, Boada J, Prat I, Portero-Otin M, Pamplona R: Cellular Dysfunction in Diabetes as Maladaptive Response to Mitochondrial Oxidative Stress. Experimental Diabetes Research. 2012.

20. Aaboe K, Krarup T, Madsbad S, Holst JJ: GLP-1: physiological effects and potential therapeutic applications. Diabetes Obesity \& Metabolism. 2008; 10:994-1003.

21. Nystrom T: The potential beneficial role of glucagon-like peptide- 1 in endothelial dysfunction and heart failure associated with insulin resistance. Hormone and Metabolic Research. 2008; 40:593-606.

22. Nystrom T, Gutniak MK, Zhang OM, Zhang F, Holst JJ, Ahren B, Sjoholm A: Effects of glucagon-like peptide-1 on endothelial function in type 2 diabetes patients with stable coronary artery disease. American Journal of Physiology-Endocrinology and Metabolism. 2004; 287:E1209-E1215.

23. Oeseburg $\mathrm{H}$, de Boer RA, Buikema $\mathrm{H}$, van der Harst $\mathrm{P}$, van Gilst $\mathrm{WH}$, Sillje HHW: Glucagon-Like Peptide 1 Prevents Reactive Oxygen Species-Induced Endothelial Cell Senescence Through the Activation of Protein Kinase A. Arteriosclerosis Thrombosis and Vascular Biology. 2010; 30:1407-U1356.

24. Scherz-Shouval R, Shvets E, Fass E, Shorer H, Gil L, Elazar Z: Reactive oxygen species are essential for autophagy and specifically regulate the activity of Atg4. Embo Journal. 2007; 26:1749-1760.

25. Lavandero S, Troncoso R, Rothermel BA, Martinet W, Sadoshima J, Hill JA: Cardiovascular autophagy Concepts, controversies, and perspectives. Autophagy. 2013; 9:1455-1466.

26. Chen YQ, Klionsky DJ: The regulation of autophagy - unanswered questions. Journal of Cell Science. 2011; 124:161-170.

27. Kiffin R, Bandyopadhyay U, Cuervo AM: Oxidative stress and autophagy. Antioxidants \& Redox Signaling. 2006; 8:152-162.

28. Russo R, Berliocchi L, Adornetto A, Varano GP, Cavaliere F, Nucci C, Rotiroti D, Morrone LA, Bagetta G, Corasaniti MT: Calpain-mediated cleavage of 
Beclin-1 and autophagy deregulation following retinal ischemic injury in vivo. Cell Death \& Disease. 2011; 2:: e144.

29. Parmigiani RB, Xu WS, Venta-Perez G, Erdjument-Bromage H, Yaneva M,

Tempst P, Marks PA: HDAC6 Is a Specific Deacetylase of Peroxiredoxins and Is Involved in Redox Regulation. Proceedings of the National Academy of Sciences of the United States of America. 2008; 105:9633-9638.

30. Valenzuela-Fernandez A, Cabrero JR, Serrador JM, Sanchez-Madrid F: HDAC6: a key regulator of cytoskeleton, cell migration and cell-cell interactions. Trends in Cell Biology. 2008; 18:291-297.

31. Kapoor KN, Barry DT, Rees RC, Dodi IA, McArdle SE, Creaser CS, Bonner PL: Estimation of peptide concentration by a modified bicinchoninic acid assay. Analytical Biochemistry. 2009; 393:138-140.

32. Brownlee M: Biochemistry and molecular cell biology of diabetic complications. Nature. 2001; 414:813-820.

33. Zhu JH, Wang KZQ, Chu CT: After the banquet Mitochondrial biogenesis, mitophagy, and cell survival. Autophagy. 2013; 9:1663-1676.

34. Williams KA, Zhang M, Xiang SY, Hu C, Wu JY, Zhang SP, Ryan M, Cox AD,

Der CJ, Fang B, et al: Extracellular Signal-regulated Kinase (ERK) Phosphorylates Histone Deacetylase 6 (HDAC6) at Serine 1035 to Stimulate Cell Migration. Journal of Biological Chemistry. 2013; 288:33156-33170.

35. Tien S C, Chang Z F. Oncogenic Shp2 disturbs microtubule regulation to cause HDAC6-dependent ERK hyperactivation. Oncogene.2013; 33:2938-46. 